\title{
Operative Torque Analysis to Evaluate Cutting Efficiency of Two Nickel-Titanium Rotary Instruments for Glide Path: An In Vitro Comparison
}

\author{
Gianluca Gambarini ${ }^{1}$, Massimo Galli ${ }^{2}$, Andrea Cicconetti ${ }^{3}$, Dario Di Nardo ${ }^{4}$, Marco Seracchiani ${ }^{5}$, Federico Valenti Obino ${ }^{6}$, \\ Gabriele Miccoli ${ }^{7}$, Luca Testarelli ${ }^{8}$
}

\begin{abstract}
Aim and objective: The aim and objective of this study was to evaluate and compare the cutting efficacy of two different nickel titanium rotary instruments by a novel methodology: the operative torque (torque and time needed to progress toward the apex).

Materials and methods: Ten fresh extracted upper first premolars with two canals were instrumented with a KaVo (Biberach, Germany) and a KaVo 1:1 handpiece at 300 rpm with maximum torque set at 2 N. One canal was instrumented with ProGlider NiTi rotary instruments (Dentsply Sirona Endodontics, Ballaigues, Switzerland), with tip size of 16.02, and the other one with EgdeGlidePath rotary instrument (EdgeEndo, Albuquerque, New Mexico). Mean instrumentation time, mean torque values, and maximum torque values were evaluated for each instrument. The significance level was set at $p<0.05$.

Results: EdgeGlidePath instruments reached the working length in significantly less time with a significantly smaller amount of torque when compared to ProGlider $(p>0.05)$. No instruments exhibited flute deformation or underwent intracanal failure.

Conclusions: Operative torque is related to the capability to cut dentin and progress toward the apex: the smaller the torque values, the higher the cutting ability (and safety). Operative torque is also dependent on debris removal and irrigation techniques. Nevertheless, both operative torque and instrumentation time are clinically relevant parameters for evaluating instruments' performance (i.e., cutting ability).

Clinical significance: Operative torque during endodontic instrumentation helps understanding the overall performance in terms of both cutting efficiency and safety.

Keywords: Cutting efficiency, Glide path, Nickel-titanium, Operative torque, Rotary instruments.

The Journal of Contemporary Dental Practice (2021): 10.5005/jp-journals-10024-3061
\end{abstract}

\section{INTRODUCTION}

The mechanical properties of nickel-titanium (NiTi) endodontic instruments have been widely investigated in the past years, including the cutting efficiency, ${ }^{1,2}$ due to their importance in affecting their clinical performance. It has been shown that because of their capability to efficiently cut and remove dentinal tissue, it is related to many instruments' features as follows: Firstly, it is dependent on design, including tip size and cross-sectional shape, number and depth of flutes, and helical and rake angles. ${ }^{3-5}$ Secondly, it is dependent on the properties of the alloy and manufacturing processes, including alloy hardness, electropolishing, heat treatments, and coating of the instruments. ${ }^{6,7}$ Finally, it is dependent on instruments' motion, including speed and torque in continuously rotating or reciprocating motions, amplitude and frequency of pecking motion, and debridement of flutes. ${ }^{8,9}$ Other differences in cutting efficiency may also be related to sterilization processes or more likely to the clinical use, which is known to reduce the sharpening of the blades.

There is no standard method to evaluate the cutting efficiency. In the last decades, it has been evaluated mainly in extracted teeth by measuring the amount of debris generated during instrumentation, by measuring the weight loss of teeth after canal preparation or by radiographically assessing the changes in canals' volumes and/or in the dentin thickness following instrumentation. Other researchers made similar evaluations using plastic blocks, while a few studies
${ }^{1-8}$ Department of Oral and Maxillo-facial Sciences, Sapienza University of Rome, Rome, Italy

Corresponding Author: Dario Di Nardo, Department of Oral and Maxillo-facial Sciences, Sapienza University of Rome, Rome, Italy, Phone: +39 3393935527, e-mail: Dario.dinardo@uniroma1.it

How to cite this article: Gambarini G, Galli M, Cicconetti A, et al. Operative Torque Analysis to Evaluate Cutting Efficiency of Two NickelTitanium Rotary Instruments for Glide Path: An In Vitro Comparison. J Contemp Dent Pract 2021;22(3):215-218.

Source of support: Nil

Conflict of interest: None

measured lateral cutting by measuring the indentation depth into a Plexiglas plate produced by a lateral pressure of the rotating $\mathrm{NiTi}$ instrument. In the majority of studies, preparation time was also recorded because a more rapid progression toward the apex was considered directly related to a better cutting efficacy. ${ }^{10-13}$

More recently, an in vivo study on operative torque was published using an innovative methodology, which proved to be a useful tool to evaluate instruments and techniques during their clinical use. ${ }^{14}$ Measuring operative torque (more precisely mean and peak torque values) allowed precise comparison of intracanal progression of different instruments, showing significant differences in both preparation time and instrumentation stresses.

() Jaypee Brothers Medical Publishers. 2021 Open Access This article is distributed under the terms of the Creative Commons Attribution 4.0 International License (https://creativecommons.org/licenses/by-nc/4.0/), which permits unrestricted use, distribution, and non-commercial reproduction in any medium, provided you give appropriate credit to the original author(s) and the source, provide a link to the Creative Commons license, and indicate if changes were made. The Creative Commons Public Domain Dedication waiver (http://creativecommons.org/publicdomain/zero/1.0/) applies to the data made available in this article, unless otherwise stated. 
The authors concluded that the device used to measure intracanal torque could be adopted to evaluate the clinical performance of instruments (including cutting efficiency) and other operatorrelated parameters, such as pecking motions or brushing motion. ${ }^{15}$ Hence, in the present study, operative torque and instrumentation time were recorded during the intracanal progression of instruments in extracted teeth as means to evaluate and compare the cutting efficiency of NiTi rotary instruments.

\section{Materials and Methods}

The whole research has been performed at the Department of Oral and Maxillo-facial Sciences of Sapienza University of Rome. Ten fresh extracted upper first premolars with two canals were selected for the study. After accessing the cavity with a 016 round bur, all canals were negotiated with a K-file size 10, and working length was established visually and radiographically. Teeth with nonpatent canals, severe canal curvatures $(>30)$, or wide or resorbed canals were excluded from the study. In each tooth, one canal was assigned to a first group and instrumented with a ProGlider NiTi rotary instrument (Dentsply Sirona Endodontics, Ballaigues, Switzerland), exhibiting tip size of 16.02 , variable progressive taper, and manufactured using m-wire alloy; the other canal was assigned to the second group and was instrumented with a EgdeGlidePath rotary instrument (EdgeEndo, Albuquerque, New Mexico). EdgeGlidePath has a triangular cross-section, with tip size of 19 and $1 \mathrm{~mm}$ maximum flute diameter (varying taper), and it is produced with FireWire heat treatment. Both instruments are meant to create a glide path using only one instrument. All teeth were prepared by the same experienced clinician, and the same amount of $5 \%$ sodium hypochlorite (Niclor, Ogna, Milan, Italy) was used in each root canal.

All nickel-titanium rotary instruments were used only in one canal. They were gently inserted with a slight pecking motion until they reached the working length and were used according to manufacturers' instructions (rotated at $300 \mathrm{rpm}$ with maximum torque set at $2 \mathrm{~N}$ ), avoiding forcing them apically. Rotary instrumentation was performed by prototype motor provided by KaVo (Biberach, Germany) and a KaVo 1:1 handpiece, as described in previous in vivo researches. ${ }^{14,15}$ All the tested instruments were able to reach the apex. During intracanal progression of the instruments, torque values were recorded automatically by the above-mentioned motor every 0.1 second (Table 1 and Fig. 1). At the same time, all measurements were

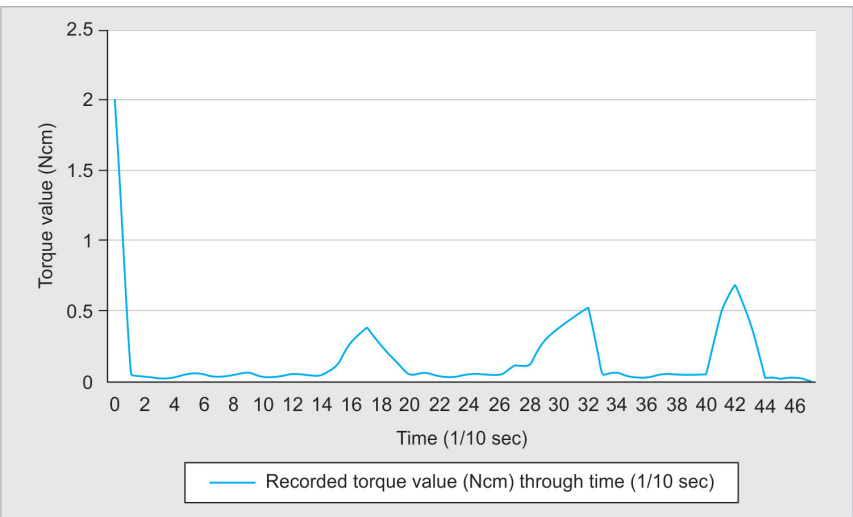

Fig. 1: Data from Table 1 are represented in a graph that shows torque trend during instrumentation
Table 1:The registered torque parameters for the first tooth negotiated with the EdgeGlidePath instrument are resumed in each column: timing (1/10 of seconds), recorded torque values $(\mathrm{N} / \mathrm{cm})$, set torque values, recorded speed values (rpm), and set speed (rpm)

\begin{tabular}{|c|c|c|c|}
\hline $1 / 10 \mathrm{sec}$ & Torque & Set torque & Set speed \\
\hline 0 & 2 & 2 & 300 \\
\hline 1 & 0.04 & 2 & 300 \\
\hline 2 & 0.03 & 2 & 300 \\
\hline 3 & 0.02 & 2 & 300 \\
\hline 4 & 0.03 & 2 & 300 \\
\hline 5 & 0.05 & 2 & 300 \\
\hline 6 & 0.04 & 2 & 300 \\
\hline 7 & 0.03 & 2 & 300 \\
\hline 8 & 0.04 & 2 & 300 \\
\hline 9 & 0.06 & 2 & 300 \\
\hline 10 & 0.03 & 2 & 300 \\
\hline 11 & 0.03 & 2 & 300 \\
\hline 12 & 0.05 & 2 & 300 \\
\hline 13 & 0.04 & 2 & 300 \\
\hline 14 & 0.04 & 2 & 300 \\
\hline 15 & 0.11 & 2 & 300 \\
\hline 16 & 0.28 & 2 & 300 \\
\hline 17 & 0.38 & 2 & 300 \\
\hline 18 & 0.25 & 2 & 300 \\
\hline 19 & 0.13 & 2 & 300 \\
\hline 20 & 0.04 & 2 & 300 \\
\hline 21 & 0.06 & 2 & 300 \\
\hline 22 & 0.03 & 2 & 300 \\
\hline 23 & 0.03 & 2 & 300 \\
\hline 24 & 0.05 & 2 & 300 \\
\hline 25 & 0.04 & 2 & 300 \\
\hline 26 & 0.04 & 2 & 300 \\
\hline 27 & 0.11 & 2 & 300 \\
\hline 28 & 0.11 & 2 & 300 \\
\hline 29 & 0.28 & 2 & 300 \\
\hline 30 & 0.38 & 2 & 300 \\
\hline 31 & 0.45 & 2 & 300 \\
\hline 32 & 0.52 & 2 & 300 \\
\hline 33 & 0.04 & 2 & 300 \\
\hline 34 & 0.06 & 2 & 300 \\
\hline 35 & 0.03 & 2 & 300 \\
\hline 36 & 0.03 & 2 & 300 \\
\hline 37 & 0.05 & 2 & 300 \\
\hline 38 & 0.04 & 2 & 300 \\
\hline 39 & 0.04 & 2 & 300 \\
\hline 40 & 0.04 & 2 & 300 \\
\hline 41 & 0.49 & 2 & 300 \\
\hline 42 & 0.69 & 2 & 300 \\
\hline 43 & 0.39 & 2 & 300 \\
\hline 44 & 0.03 & 2 & 300 \\
\hline 45 & 0.02 & 2 & 300 \\
\hline 46 & 0.03 & 2 & 300 \\
\hline 47 & 0 & 2 & 300 \\
\hline
\end{tabular}


saved on a memory card, and an Excel file was used to calculate the mean instrumentation time, mean torque values, and maximum torque values. For all tested instruments, the following parameters were analyzed statistically using one-way analysis of variance (ANOVA) test (IBM Corp. Released 2013. IBM SPSS Statistics for Windows, Version 22.0. Armonk, New York:IBM Corp.): mean instrumentation time, mean torque values, and maximum torque values. The significance level was set at $p<0.05$. Visible signs of instrument deformation or fracture, if any, were recorded.

\section{Results}

Table 2 shows mean torque values recorded in the two different canals, and instrumentation meantime. Data were normally distributed (Shapiro-Wilk's test, $p>0.05$ ), and there was homogeneity of variance (Levene's test, $p>0.05$ ). One-way ANOVA revealed a significant difference between the groups regarding the mean and maximum (peak) torque $(p<0.05)$. There was also a significant difference in mean instrumentation time $(p<0.05)$. Overall, the EdgeGlidePath instruments reached the working length in significantly less time with a significantly smaller amount of torque when compared to ProGlider. No instrument exhibited flute deformation or underwent intracanal failure.

Table 2: Comparison between the two instruments

\begin{tabular}{lllllll}
\hline & & \multicolumn{2}{c}{ ProGlider } & & \multicolumn{2}{c}{ EdgeGlidePath } \\
& $n$ & Mean & SD & & Mean & SD \\
\hline Mean torque & 10 & 0.41 & 0.21 & & $0.25 \mathrm{a}$ & 0.19 \\
Maximum torque & 10 & 1.56 & 0.53 & $1.21 \mathrm{~b}$ & 0.27 \\
$\begin{array}{l}\text { Mean instrumentation } \\
\text { time, speed }\end{array}$ & 10 & 14.87 & 5.49 & $9.25 \mathrm{C}$ & 2.58 \\
\hline
\end{tabular}

Mean torque values and mean instrumentation time recorded during intracanal progression to the apex with the two tested instruments in the two canals of the same extracted tooth. The highest registered torque value (max torque) is also displayed. Different superscript letter indicates statistical significance $(p<0.05)$

\section{Discussion}

Glide path instruments were selected for this study because they are the first NiTi rotary instruments used by many clinicians, and their performance is not affected by previous instrumentation. Results showed a significant difference between the two instruments for all the parameters tested: mean instrumentation time, mean torque values, and maximum torque values. Since all instruments were operated in similar canals with the same parameters (speed, torque, and motions), differences could be related to different design, manufacturing process, or dimensions. The proposed innovative methodology (measurements of operative torque) allowed detailed visualization and excellent comparison of instrument progression toward the apex, showing detectable differences in both time and torque values. In addition, less instrumentation torque means lower stress during intracanal instrumentation, thus increasing not only performance (due to increased cutting ability) but also safety.

The two tested glide path instruments have a similar tip size (size 16 vs size 19). In a lateral cutting test, usually the greater the diameters, the greater is the cutting efficiency. In the present study, cutting efficiency was assessed by measuring the operative torque, if more efficient instrument needs less torque and less time to progress and reach the apex. It has been demonstrated that instrument design and manufacturing play a role ${ }^{3}$ in determining performance; for example, wider cutting blades contacting more dentine on the canal wall increase the lateral resistance. Heat treatments may produce a more ductile alloy, with increased deformation of the cutting blades and greater contact with dentinal walls. All these factors may increase the torque generated during intracanal instrumentation.

Instruments with greater tapers and dimensions need more operative torque to cut and progress toward the apex, avoiding the taper lock effect in their middle and coronal parts. ${ }^{16}$ Therefore, the lower torque values required by EdgeGlidePath instruments are probably related to one or all these factors: design, manufacturing process (including heat treatment), and taper. It is difficult to precisely analyze the influence of each of the above-mentioned factors because both heat treatments and tapers are not clearly disclosed by manufacturers. ${ }^{17-19}$

It is not easy to correlate data from the present study with similar studies because the instruments are different, in terms of design, dimensions, and clinical use; moreover, the proposed novel methodology is very different from the in vitro tests performed in other studies. ${ }^{10-13}$ More specifically, the proposed novel methodology is closer to the real clinical use of the instruments, and with some approximation, it could be compared with studies evaluating instrumentation time. Indeed, studies about instrumentation time are difficult to compare because they are highly influenced by variations in canal anatomy, curvatures, canal diameters, hardness of dentine, etc. Differences in the latter parameter (surface hardness), however, are common limitations in all cutting tests; this can probably explain why there is no international approved standard test for cutting efficiency.

Operative torque is also related to the capability to remove debris because debris entrapped among flutes can increase torque and reduce cutting ability. A possible limitation of the present test is because of the fact that operative torque is assessing cutting efficiency not exclusively since it is also dependent on flutes' debris removal and irrigation techniques. Moreover, it is a comprehensive test, which cannot distinguish which part of the instrument is efficient in cutting; the latter evaluation could be useful to test in vitro modifications in the instruments' design. Nevertheless, both operative torque and instrumentation time are clinically relevant parameters because they evaluate performance in a way very similar to the clinical use of the instruments. ${ }^{20-22}$

\section{Conclusion}

Under the experimental conditions of the present study, EdgeGlidePath instruments reached the working length in significantly less time with a significantly smaller amount of torque when compared to ProGlider, thus exhibiting an overall better cutting efficiency, with less risk of overstressing the instruments. The instruments were similar in their clinical use and dimensions but different in design and manufacturing. However, it was not possible to assess which factor (design, manufacturing process, or dimensions) played a major role in determining these results. Future studies, by comparing instruments with only one parameter different, may help to precisely assess the role of each parameter in determining cutting efficiency. 


\section{Clinical Significance}

Operative torque during endodontic instrumentation helps in understanding overall performance in terms of both cutting efficiency and safety.

\section{References}

1. Plotino G, Giansiracusa Rubini A, Grande NM, et al. Cutting efficiency of Reciproc and WaveOne reciprocating instruments. J Endod 2014;40(8):1228-1230. DOI: 10.1016/j.joen.2014.01.041.

2. Gambarini G, Giansiracusa Rubini A, Sannino G, et al. Cutting efficiency of nickel-titanium rotary and reciprocating instruments after prolonged use. Odontology 2016;104(1):77-81. DOI: 10.1007/ s10266-014-0183-0.

3. Baek SH, Lee CJ, Versluis A, et al. Comparison of torsional stiffness of nickel-titanium rotary files with different geometric characteristics. J Endod 2011;37:1283-1286. DOI: 10.1016/j.joen.2011.05.032.

4. Blum J, Machtou P, Micallef J. Location of contact areas on rotary Profile instruments in relationship to the forces developed during mechanical preparation on extracted teeth. Int Endod J 1999;32(2):108-114. DOI: 10.1046/j.1365-2591.1999.00200.x.

5. Gambarini G, Miccoli G, Seracchiani M, et al. Role of the flat-designed surface in improving the cyclic fatigue resistance of endodontic NiTi rotary instruments. Materials (Basel) 2019;12(16):2523. DOI: 10.3390/ ma12162523.

6. Gambarini G, Galli M, Di Nardo D, et al. Differences in cyclic fatigue lifespan between two different heat treated NiTi endodontic rotary instruments: WaveOne Gold vs EdgeOne Fire. J Clin Exp Dent 2019;11(7):e609-e613. DOI: 10.4317/jced.55839.

7. Miccoli G, Gaimari G, Seracchiani M, et al. In vitro resistance to fracture of two nickel-titanium rotary instruments made with different thermal treatments. Ann Stomatol (Roma) 2017;8(2):53-58. DOI: 10.11138/ads/2017.8.2.059.

8. Gambarini G, Piasecki L, Miccoli G, et al. Classification and cyclic fatigue evaluation of new kinematics for endodontic instruments. Aust Endod J 2019;45(2):154-162. DOI: 10.1111/aej.12294.

9. Gambarini G, Di Nardo D, Miccoli G, et al. The influence of a new clinical motion for endodontic instruments on the incidence of postoperative pain. Clin Ter 2017;168(1):e23-e27. DOI: 10.7417/ CT.2017.1977.

10. Pedullà E, Leanza G, La Rosa GRM, et al. Cutting efficiency of conventional and heat-treated nickel-titanium rotary or reciprocating glide path instruments. Int Endod J 2020;53(3):376-384. DOI: 10.1111/ iej.13224.

11. de Cristofaro Almeida G, Aun DP, Resende PD, et al. Comparative analysis of torque and apical force to assess the cutting behaviour of ProTaper Next and ProTaper Universal endodontic instruments. Aust Endod J 2020;46(1):52-59. DOI: 10.1111/aej.12351.

12. Spicciarelli V, Corsentino G, Ounsi HF, et al. Shaping effectiveness and surface topography of reciprocating files after multiple simulated uses. J Oral Sci 2019;61(1):45-52. DOI: 10.2334/josnusd.17-0311.

13. Vasconcelos RA, Arias A, Peters OA. Lateral and axial cutting efficiency of instruments manufactured with conventional nickel-titanium and novel gold metallurgy. Int Endod J 2018;51(5):577-583. DOI: 10.1111/ iej.12822.

14. Gambarini G, Seracchiani M, Piasecki L, et al. Measurement of torque generated during intracanal instrumentation in vivo. Int Endod J 2019;52(5):737-745. DOI: 10.1111/iej.13042.

15. Gambarini G, Galli M, Seracchiani M, et al. In vivo evaluation of operative torque generated by two nickel-titanium rotary instruments during root canal preparation. Eur J Dent 2019;13(4):556562. DOI: 10.1055/s-0039-1698369.

16. Sattapan B, Palamara JE, Messer HH. Torque during canal instrumentation using rotary nickel-titanium files. J Endod 2000;26(3):156-160. DOI: 10.1097/00004770-200003000-00007.

17. Boessler C, Peters $\mathrm{O}$, Zehnder M. Impact of lubricant parameters on rotary instrument torque and force. J Endod 2007;33(3):280-283. DOI: 10.1016/j.joen.2006.11.007.

18. Boessler C, Paque F, Peters OA. The effect of electropolishing on torque and force during simulated root canal preparation with ProTaper shaping files. J Endod 2009;35(1):102-106. DOI: 10.1016/j. joen.2008.09.008.

19. Kwak, SW, Ha JH, Cheung GS, et al. Effect of the glide path establishment on the torque generation to the files during instrumentation: an in vitro measurement. J Endod 2018;44(3):496500. DOI: 10.1016/j.joen.2017.09.016.

20. Peters O, Barbakow F. Dynamic torque and apical forces of Profile. 04 rotary instruments during preparation of curved canals. Int Endod J 2002;35(4):379-389. DOI: 10.1046/j.0143-2885.2001.00494.x.

21. Schrader C, Peters OA. Analysis of torque and force with differently tapered rotary endodontic instruments in vitro. J Endod 2005;31(2):120-123. DOI: 10.1097/01.don.0000137634.20499.1d.

22. Yared G, Bou Dagher FE, Machtou P. Influence of rotational speed, torque and operator's proficiency on Profile failures. Int Endod J 2001;34(1):47-53. DOI: 10.1046/j.1365-2591.2001.00352.x. 